PROCEEDINGS OF THE

AMERICAN MATHEMATICAL SOCIETY

Volume 139, Number 3, March 2011, Pages 909-916

S 0002-9939(2010)10333-7

Article electronically published on October 22, 2010

\title{
REMARKS ON THE AREA THEOREM IN THE THEORY OF UNIVALENT FUNCTIONS
}

\author{
M. PAVLOVIĆ AND JOSÉ ÁNGEL PELÁEZ
}

(Communicated by Nigel J. Kalton)

\begin{abstract}
We prove an integral extension of the classical area theorem for univalent functions. We give an application finding geometric conditions on the image domain of a univalent function $f$ which imply that $f$ belongs to the Hardy space $H^{p}, 0<p<\infty$.
\end{abstract}

\section{INTRODUCTION AND MAIN RESULTS}

Let $\mathbb{D}$ denote the open unit disk of the complex plane $\mathbb{C}$. A complex-valued function defined in $\mathbb{D}$ is said to be univalent if it is analytic and one-to-one there. We refer to [5] and [8] for the theory of these functions. Throughout the paper, $\mathcal{U}$ will stand for the class of all univalent functions in $\mathbb{D}$. The classical area theorem [5. p. 29], which is a key in the proof of a good number of results in the theory of univalent functions, can be stated as follows:

Theorem 1. If $\varphi(z)=\sum_{n=0}^{\infty} b_{n} z^{n}, b_{0} \neq 0$, is an analytic function in $\mathbb{D}$ such that the meromorphic function $\varphi(z) / z$ is one-to-one in $\mathbb{D}$, then

$$
\sum_{n=1}^{\infty} \frac{n^{2}\left|b_{n}\right|^{2}}{n+1} \leq \sum_{n=0}^{\infty} \frac{\left|b_{n}\right|^{2}}{n+1}
$$

or, equivalently,

$$
\int_{\mathbb{D}}\left|z \varphi^{\prime}(z)\right|^{2} d m(z) \leq \int_{\mathbb{D}}|\varphi(z)|^{2} d m(z) .
$$

Here, $d m(z)=d x d y$ denotes the usual Lebesgue area measure.

In this paper we generalize Theorem 1 in the following way.

Theorem 2. Let $p>0$. If $\varphi$ is a function as in Theorem 1 and $\varphi(z) \neq 0$ for all $z \in \mathbb{D}$, then

$$
\int_{\mathbb{D}}|z|^{p}|\varphi(z)|^{p-2}\left|\varphi^{\prime}(z)\right|^{2} d m(z) \leq \int_{\mathbb{D}}|z|^{p-2}|\varphi(z)|^{p} d m(z) .
$$

Received by the editors October 5, 2009 and, in revised form, December 15, 2009.

2010 Mathematics Subject Classification. Primary 30C55, 30H10.

Key words and phrases. Area theorem, univalent functions, Hardy spaces.

The first author is supported by MNZŽS Serbia, Project ON144010.

The second author is partially supported by the Ramón y Cajal program of MICINN (Spain), the Spanish (Grants MTM2007-60854 and MTM2006-26627) and regional Andalusian (Grants FQM210 and P06-FQM01504) governments. 
Consequently, we deduce the following theorem on univalent functions.

Theorem 3. If $f$ is univalent in $\mathbb{D}, f(0)=0$ and $p>0$, then

$$
\int_{\mathbb{D}}|z|^{2 p-2}|f(z)|^{-p}\left|1-\frac{z f^{\prime}(z)}{f(z)}\right|^{2} d m(z) \leq \int_{\mathbb{D}}|z|^{2 p-2}|f(z)|^{-p} d m(z)
$$

or, equivalently,

$$
\int_{\mathbb{D}}|z|^{2 p-2}|f(z)|^{-p}\left|\frac{z f^{\prime}(z)}{f(z)}\right|^{2} d m(z) \leq 2 \int_{\mathbb{D}}|z|^{2 p-2}|f(z)|^{-p} \operatorname{Re}\left(\frac{z f^{\prime}(z)}{f(z)}\right) d m(z) .
$$

Given a space $X$ of analytic functions, one of the most interesting problems in the theory of univalent functions is finding geometric conditions on a domain $\Omega$ which imply that $\Omega$ is an $X$-domain; that is, any analytic function $f$ defined on $\mathbb{D}$ with $f(\mathbb{D}) \subset \Omega$ belongs to $X$. This problem has been solved for a good number of spaces, such as the Bloch space, Besov spaces $B^{p}, 1<p<\infty, \ldots$ (see [1, 3]). However, this is an open problem for $H^{p}$ spaces (see 44). We shall use Theorem 3 to find geometric conditions on the image domain of a function $f \in \mathcal{U}$ which imply its membership in $H^{p}$. For simplicity, we shall assume that $0 \in f(\mathbb{D})$.

Given a domain $\Omega \subset \mathbb{C}$ and a point $w$ in $\Omega$, we shall write $d_{\Omega}(w)$ to denote the (Euclidean) distance from $w$ to the boundary $\partial \Omega$. The following inequalities play an essential role in the proof of our results (see, e.g., [9], Corollary 1.4).

If $\Omega$ is a simply connected proper subdomain of $\mathbb{C}$ and if $F$ is a conformal mapping from $\Delta$ onto $\Omega$, then we have

$$
d_{\Omega}(F(z)) \leq\left|F^{\prime}(z)\right|\left(1-|z|^{2}\right) \leq 4 d_{\Omega}(F(z)), \quad z \in \mathbb{D} .
$$

The following result is proved in [2, Corollary 7].

Corollary 1. Suppose that $1 / 2 \leq p<\infty$ and that $f \in A^{2 p} \cap \mathcal{U}$. Set $\Omega=f(\mathbb{D})$ and suppose that $0 \in \Omega$. For $\varepsilon>0$, set $\Omega_{\varepsilon}=\{w \in \Omega:|w|>\varepsilon\}$. If

$$
\int_{\Omega_{\varepsilon}} \frac{d_{\Omega}(w)^{2 p-2}}{|w|^{2 p}} d m(w)<\infty
$$

for all sufficiently small $\varepsilon>0$, then $f \in H^{p}$.

Here, we shall prove the following extension of this result.

Theorem 4. Suppose that $0<\beta<1,1-\frac{\beta}{2}<p<\infty$, and $f \in A^{2 p} \cap \mathcal{U}$. Set $\Omega=f(\mathbb{D})$ and suppose that $0 \in \Omega$. If

$$
\int_{\Omega_{\varepsilon}} \frac{d_{\Omega}(w)^{\frac{2 p-2}{\beta}}}{|w|^{\frac{2 p-2}{\beta}+2 \frac{\beta-\delta}{\beta}}} d m(w)<\infty
$$

for some $\delta, 0<\delta<\frac{1+p}{2}$ and all sufficiently small $\varepsilon>0$, then $f \in H^{p}$.

Moreover, we shall prove that this result is sharp in a certain sense.

The paper is organized as follows. Section 2 is devoted to proving Theorem 2 and Theorem 3. Corollary 4 and some other results are proved in section 3 .

\section{Proof of the main Results}

The proof of Theorem 2 is based on the following fact due to Prawitz [10]. The proof is borrowed from [7] (see also [6]). 
Theorem 5. Let $f: \mathbb{D} \mapsto \mathbb{C}$ be a univalent function and $f(0)=0$, and let

$$
J_{p}(r)=J_{p}(r, f)=\frac{1}{2 \pi} \int_{0}^{2 \pi}\left|f\left(r e^{i \theta}\right)\right|^{-p} d \theta, \quad p>0, \quad 0<r<1 .
$$

Then

$$
\begin{array}{rlr}
2 \pi J_{p}^{\prime}(r) & =-(p / r) \operatorname{Im} \int_{\Gamma_{r}}|w|^{-p-2} \bar{w} d w \quad(w=u+i v) \\
& =-(p / r) \int_{\Gamma_{r}}|w|^{-p-2}(u d v-v d u) \\
& <0,
\end{array}
$$

for all $r \in(0,1)$, where $\Gamma_{r}$ is the image under $f$ of the circle $\{\zeta \in \mathbb{C}:|\zeta|=r\}$ and $\Gamma_{r}$ is positively oriented.

Proof. We have

$$
\begin{array}{rlr}
2 \pi J_{p}^{\prime}(r) & =-p \int_{0}^{2 \pi}\left|f\left(r e^{i \theta}\right)\right|^{-p-2} \operatorname{Re}\left\{\overline{f\left(r e^{i \theta}\right)} f^{\prime}\left(r e^{i \theta}\right) e^{i \theta}\right\} d \theta \\
& =-(p / r) \operatorname{Im} \int_{|\zeta|=r}|f(\zeta)|^{-p-2} \overline{f(\zeta)} f^{\prime}(\zeta) d \zeta & \\
& =-(p / r) \operatorname{Im} \int_{\Gamma_{r}}|w|^{-p-2} \bar{w} d w & (w=u+i v) \\
& =-(p / r) \int_{\Gamma_{r}}|w|^{-p-2}(u d v-v d u),
\end{array}
$$

where $\Gamma_{r}$ is the image under $f$ of the circle $|\zeta|=r$ and the curve $\Gamma_{r}$ is positively oriented. Now we apply Green's formula to the domain $\Omega_{r, R}$ bounded by $\Gamma_{r}$ and the circle $|w|=R$, where $R>\max _{|z|=r}|f(z)|$. Since

$$
\partial\left(|w|^{-p-2} u\right) / \partial u-\partial\left(-|w|^{-p-2} v\right) / \partial v=-p|w|^{-p-2},
$$

we have

$$
\begin{aligned}
\int_{|w|=R}|w|^{-p-2}(u d v-v d u)-\int_{\Gamma_{r}}|w|^{-p-2}(u d v-v d u) \\
=-p \iint_{\Omega_{r, R}}|w|^{-p-2} d u d v .
\end{aligned}
$$

The first integral is equal to $2 \pi R^{-p}$, and therefore

$$
J_{p}^{\prime}(r)=-(p / r) R^{-p}-\left(p^{2} / 2 \pi r\right) \iint_{\Omega_{r, R}}|w|^{-p-2} d u d v .
$$

Letting $R$ tend to $\infty$ we get

$$
J_{p}^{\prime}(r)=-\left(p^{2} / 2 \pi r\right) \iint_{\Omega_{r}}|w|^{-p-2} d u d v,
$$

where $\Omega_{r}$ is the exterior of the curve $\Gamma_{r}$. This concludes the proof.

Proof of Theorem 2. With the hypotheses of Theorem 2, let $g(z)=\varphi(z) / z$ and $f(z)=1 / g(z)=z / \varphi(z)$. Let

$$
I_{p}(r)=I_{p}(r, g)=\frac{1}{2 \pi} \int_{0}^{2 \pi}\left|g\left(r e^{i \theta}\right)\right|^{p} d \theta=J_{p}(r, f), \quad p>0 .
$$


The function $f$ satisfies the hypotheses of Theorem [5, and therefore

$$
2 \pi I_{p}^{\prime}(r)=2 \pi J_{p}^{\prime}(r)=-(p / r) \int_{\Gamma_{r}}|w|^{-p-2}(u d v-v d u)<0 .
$$

By the change $w \mapsto 1 / w$ we get

$$
2 \pi I_{p}^{\prime}(r)=(p / r) \int_{\gamma_{r}}|w|^{p-2}(u d v-v d u)<0,
$$

where $\gamma_{r}$ denotes the curve $w=g\left(r e^{i t}\right), 0 \leq t \leq 2 \pi$, which is a curve of negative orientation. Now we try to parameterize $\gamma_{r}$ by $w=F\left(e^{i t}\right)$, where $F\left(e^{i t}\right) \equiv g\left(r e^{i t}\right)$; indeed we choose

$$
F(z)=\frac{\bar{z}}{r} \varphi(r z)
$$

Then we have

$$
\int_{\gamma_{r}}|w|^{p-2}(u d v-v d u)=\operatorname{Im} \int_{|\zeta|=1}|F(\zeta)|^{p-2} \overline{F(\zeta)} d F(\zeta) .
$$

Now, we choose a circle $T_{\rho}$ of radius $\rho$ centered at $0\left(0<\rho<\frac{1}{2}\right)$ and apply Green's formula to the annulus $A_{\rho}:=\{z: \rho \leq|z| \leq 1\}$ to get

$$
\operatorname{Im} \int_{|\zeta|=1}|F(\zeta)|^{p-2} \overline{F(\zeta)} d F(\zeta)=p \int_{A_{\rho}}|F|^{p-2} J_{F} d m+\operatorname{Im} \int_{T \rho}|F|^{p-2} \bar{F} d F,
$$

where $J_{F}(z)$ is the Jacobian of $F$,

$$
\begin{aligned}
J_{F}(z) & =|\partial F / \partial z|^{2}-|\partial F / \partial \bar{z}|^{2} \\
& =\left|z \varphi^{\prime}(r z)\right|^{2}-|\varphi(r z)|^{2} / r^{2} ;
\end{aligned}
$$

here the circles are positively oriented. From the properties of $\varphi$ it follows that

$$
\left.\left|\operatorname{Im} \int_{T \rho}\right| F\right|^{p-2} \bar{F} d F \mid \leq C \rho^{p}
$$

and

$$
\int_{\mathbb{D}}|F|^{p-2}\left|J_{F}\right| d m \leq C \int_{\mathbb{D}}|z|^{p-2} d m(z)<\infty .
$$

Hence, by passing to the limit as $\rho \rightarrow 0$, we get

$$
\operatorname{Im} \int_{|\zeta|=1}|F(\zeta)|^{p-2} \overline{F(\zeta)} d F(\zeta)=p \int_{\mathbb{D}}|F|^{p-2} J_{F} d m .
$$

From this, (2.1) and (2.2), it follows that

$$
\int_{\mathbb{D}}|F(z)|^{p-2} J_{F}(z) d m(z)<0 ;
$$

that is,

$$
\begin{aligned}
\int_{\mathbb{D}}|z \varphi(r z)|^{p-2}\left|z \varphi^{\prime}(r z)\right|^{2} d m(z)<\left.r^{-2} \int_{\mathbb{D}}|z|^{p-2} \varphi(r z)\right|^{p} d m(z) \\
=2 \pi r^{-2} \int_{0}^{1} t^{p-1} I_{p}(r t, \varphi) d t
\end{aligned}
$$

where, as above,

$$
I_{p}(s, \varphi)=\frac{1}{2 \pi} \int_{0}^{2 \pi}\left|\varphi\left(s e^{i \theta}\right)\right|^{p} d \theta .
$$


As is well known, $I_{p}(s, \varphi)$ increases with $s$ and so we can apply the monotone convergence theorem to get

$$
\begin{aligned}
\lim _{r \rightarrow 1^{-}} \int_{0}^{1} t^{p-1} I_{p}(r t, \varphi) d t & =\int_{0}^{1} t^{p-1} \lim _{r \rightarrow 1^{-}} I_{p}(r t, \varphi) d t \\
& =\int_{0}^{1} t^{p-1} I_{p}(t, \varphi) d t .
\end{aligned}
$$

From this and (2.3), via Fatou's lemma, we obtain (1.1). This completes the proof of Theorem 2 .

Proof of Theorem 3. If $f$ is univalent in $\mathbb{D}, f(0)=0$, then let $\varphi(z)=z / f(z)$. Since

$$
\varphi^{\prime}(z)=\frac{f(z)-z f^{\prime}(z)}{f(z)^{2}}
$$

we see, by Theorem 2 , that

$$
\int_{\mathbb{D}}|z|^{2 p-2}|f(z)|^{2-p}\left|\frac{f(z)-z f^{\prime}(z)}{f(z)^{2}}\right|^{2} d m(z) \leq \int_{\mathbb{D}}|z|^{p-2}\left|\frac{z}{f(z)}\right|^{p} d m(z) .
$$

The result follows.

Remark 1. Equality is possible in (1.1), (1.2) and (1.3). In the case of (1.1) we take

$$
\varphi(z)=\left(1-e^{-i \theta} z\right)^{2}, \quad \theta \in[0,2 \pi] .
$$

Then

$$
\operatorname{Im} \int_{|\zeta|=1}|F(\zeta)|^{p-2} \overline{F(\zeta)} d F(\zeta)=0
$$

where

$$
F(z)=\bar{z} \varphi(z)=\bar{z}\left(1-e^{-i \theta} z\right)^{2} .
$$

Now an application of Green's formula as above shows that

$$
\int_{\mathbb{D}}|F|^{p-2} J_{F} d m=0
$$

which implies (1.1).

The above deduction of Theorem 3 from Theorem 2 shows that equality in (1.2) and (1.3) is attained if $f_{\theta}$ is any rotation of Koebe's function:

$$
f_{\theta}(z)=\frac{z}{\left(1-e^{-i \theta} z\right)^{2}} \text {. }
$$

\section{Applications}

First, we present the following simple but useful lemma.

Lemma 1. If $f$ is univalent in $\mathbb{D}, f(0)=0$ and $p>0$, then

$$
\int_{\mathbb{D}}|z|^{2 p}|f(z)|^{-p}\left|\frac{f^{\prime}(z)}{f(z)}\right|^{2} d m(z)<\infty
$$


Proof. Observe that if $f \in \mathcal{U}$ with $f(0)=0$, then

$$
\int_{\mathbb{D}}|z|^{2 p-2}|f(z)|^{-p} d m(z)<\infty \text { for any } p>0,
$$

which together with Theorem 3 gives that

$$
\begin{aligned}
& \int_{\mathbb{D}}|z|^{2 p}|f(z)|^{-p}\left|\frac{f^{\prime}(z)}{f(z)}\right|^{2} d m(z) \\
& \leq 2 \int_{\mathbb{D}}|z|^{2 p-2}|f(z)|^{-p}\left|1-\frac{z f^{\prime}(z)}{f(z)}\right|^{2} d m(z)+2 \int_{\mathbb{D}}|z|^{2 p-2}|f(z)|^{-p} d m(z) \\
& \leq 4 \int_{\mathbb{D}}|z|^{2 p-2}|f(z)|^{-p} d m(z)<\infty .
\end{aligned}
$$

This finishes the proof.

Now, we are ready to obtain our result on $H^{p}$-univalent functions.

Proof of Theorem 4. Bearing in mind [2, Theorem 1], it is enough to prove that

$$
\int_{\mathbb{D}}\left|f^{\prime}(z)\right|^{p}\left(1-|z|^{2}\right)^{p-1} d m(z)<\infty
$$

Take $\eta>0$ such that $\{|w|<\eta\} \subset \Omega$ and take $\varepsilon$ with $0<\varepsilon<\eta$ and set $\mathbb{D}_{\varepsilon}=f^{-1}\left(\Omega_{\varepsilon}\right)$.

By Hölder's inequality (with conjugate exponents $\frac{2}{1+\beta}>1$ and $\frac{2}{1-\beta}$ ) and Lemma 1, we deduce that

$$
\begin{aligned}
& \int_{\mathbb{D}_{\varepsilon}}\left|f^{\prime}(z)\right|^{p}\left(1-|z|^{2}\right)^{p-1} d m(z) \\
& =\int_{\mathbb{D}_{\varepsilon}}\left[\left(1-|z|^{2}\right)\left|f^{\prime}(z)\right|\right]^{p-1}\left|f^{\prime}(z)\right|^{\beta}|z|^{-2 \delta}|f(z)|^{1-\beta+\delta}\left[|f(z)|^{-\delta}|z|^{2 \delta}\left|\frac{f^{\prime}(z)}{f(z)}\right|^{1-\beta}\right] d m(z) \\
& \leq\left(\int_{\mathbb{D}_{\varepsilon}}\left[\left(1-|z|^{2}\right)\left|f^{\prime}(z)\right|\right]^{\frac{2(p-1)}{1+\beta}}\left|f^{\prime}(z)\right|^{\frac{2 \beta}{1+\beta}}|z|^{-\frac{4 \delta}{1+\beta}}|f(z)|^{\frac{2(1-\beta+\delta)}{1+\beta}} d m(z)\right)^{\frac{1+\beta}{2}} \\
& \quad \times\left(\int_{\mathbb{D}_{\varepsilon}}|f(z)|^{-\frac{2 \delta}{1-\beta}}|z|^{\frac{4 \delta}{1-\beta}}\left|\frac{f^{\prime}(z)}{f(z)}\right|^{2} d m(z)\right)^{\frac{1-\beta}{2}} \\
& \leq C\left(\int_{\mathbb{D}_{\varepsilon}}\left[\left(1-|z|^{2}\right)\left|f^{\prime}(z)\right|\right]^{\frac{2(p-1)}{1+\beta}}\left|f^{\prime}(z)\right|^{\frac{2 \beta}{1+\beta}}|z|^{-\frac{4 \delta}{1+\beta}}|f(z)|^{\frac{2(1-\beta+\delta)}{1+\beta}} d m(z)\right)^{\frac{1+\beta}{2}} .
\end{aligned}
$$

On the other hand, if $0<\delta<\frac{1+p}{2}$, using Hölder's inequality (with conjugate exponents $\frac{1+\beta}{\beta}$ and $\left.1+\beta\right)$, making the change of variable $w=f(z)$ and bearing in 
mind (1.4) and that $f \in A^{2 p}$, we deduce that

$$
\begin{aligned}
& \int_{\mathbb{D}_{\varepsilon}}\left[\left(1-|z|^{2}\right)\left|f^{\prime}(z)\right|\right]^{\frac{2(p-1)}{1+\beta}}\left|f^{\prime}(z)\right|^{\frac{2 \beta}{1+\beta}}|z|^{-\frac{4 \delta}{1+\beta}}|f(z)|^{\frac{2(1-\beta+\delta)}{1+\beta}} d m(z) \\
& =\int_{\mathbb{D}_{\varepsilon}} \frac{\left[\left(1-|z|^{2}\right)\left|f^{\prime}(z)\right|\right]^{\frac{2(p-1)}{1+\beta}}}{|f(z)|^{\frac{2(p+\beta-1-\delta)}{1+\beta}}}\left|f^{\prime}(z)\right|^{\frac{2 \beta}{1+\beta}}|z|^{-\frac{4 \delta}{1+\beta}} \mid f(z) \frac{\frac{2 p}{1+\beta}}{1+\beta} d m(z) \\
& =\left(\int_{\mathbb{D}_{\varepsilon}} \frac{\left[\left(1-|z|^{2}\right)\left|f^{\prime}(z)\right|\right]^{\frac{2(p-1)}{\beta}}}{|f(z)|^{\frac{2(p+\beta-1-\delta)}{\beta}}}\left|f^{\prime}(z)\right|^{2} d m(z)\right)^{\frac{\beta}{1+\beta}} \\
& \quad \times\left(\int_{\mathbb{D}}|z|^{-4 \delta}|f(z)|^{2 p} d m(z)\right)^{\frac{1}{1+\beta}} \\
& \leq C\left(\int_{\Omega_{\varepsilon}} \frac{d_{\Omega}(w)^{\frac{2 p-2}{\beta}}}{|w|^{\frac{2 p-2}{\beta}+2 \frac{\beta-\delta}{\beta}}} d m(w)\right)^{\frac{\beta}{1+\beta}}<\infty
\end{aligned}
$$

which, together with (3.1) and (3.2), finishes the proof.

Moreover, we are able to prove that this result is sharp in the following sense.

Theorem 6. If $0<\beta<1$ and $1-\frac{\beta}{2}<p<\infty$, then there exists a univalent function $g \in A^{2 p} \backslash H^{p}$ with $g(0)=0$ and such that, setting $\Omega=g(\mathbb{D})$ and $\Omega_{\varepsilon}=$ $\{w \in \Omega:|w|>\varepsilon\}$,

$$
\int_{\Omega_{\varepsilon}} \frac{d_{\Omega}(w)^{\frac{2 p-2}{\beta}}}{|w|^{\frac{2 p-2}{\beta}}+2+\kappa} d m(w)<\infty, \quad \varepsilon>0
$$

for every $\kappa>0$.

Proof of Theorem 6. We shall follow the lines of the proof of [2, Theorem 8]. Take $p \in(1 / 2, \infty)$ and let $f$ be the function defined in the proof of [2, Theorem 3], that is,

Set

$$
f(z)=\left[\frac{1}{(1-z) \log \frac{2 e}{1-z}}\right]^{\frac{1}{p}}, \quad z \in \mathbb{D} .
$$

$$
g(z)=f(z)-f(0), \quad z \in \mathbb{D} .
$$

Then $g$ is univalent, $g(0)=0$ and $g \in A^{2 p} \backslash H^{p}$. Finally, we shall see that (3.3) holds.

Take $\varepsilon>0$. Since $g(0)=0$, there exists $\eta$ with $0<\eta<1$ such that

$$
g^{-1}\left(\Omega_{\varepsilon}\right) \subset \mathbb{D}_{\eta}=\{z \in \mathbb{D}:|z|>\eta\} .
$$

We have that

$$
g^{\prime}(z)=\frac{1}{p(1-z)^{1+\frac{1}{p}}}\left[\left(\frac{1}{\log \frac{2 e}{1-z}}\right)^{\frac{1}{p}}\left(1-\frac{1}{\log \frac{2 e}{1-z}}\right)\right], \quad z \in \mathbb{D}
$$

and that there exists a positive constant $C$ such that

$$
|g(z)| \geq C\left|\frac{1}{(1-z) \log \frac{2 e}{1-z}}\right|^{\frac{1}{p}} \quad z \in \mathbb{D}_{\eta} .
$$


So, using (1.4) and assuming without loss of generality that $\kappa<p\left(\frac{2 p-2}{\beta}+1\right)$, we obtain

$$
\begin{aligned}
& \int_{\Omega_{\varepsilon}} \frac{d_{\Omega}(w)^{\frac{2 p-2}{\beta}}}{|w|^{\frac{2 p-2}{\beta}+2+\kappa}} d m(w) \\
& \leq C \int_{\mathbb{D}_{\eta}}\left(1-|z|^{2}\right)^{\frac{2 p-2}{\beta}} \frac{\left|g^{\prime}(z)\right|^{\frac{2 p-2}{\beta}+2}}{|g(z)|^{\frac{2 p-2}{\beta}+2+\kappa}} d m(z) \\
& \leq C \int_{\mathbb{D}_{\eta}}\left(1-|z|^{2}\right)^{\frac{2 p-2}{\beta}} \frac{\left|(1-z) \log \frac{2 e}{1-z}\right|^{\frac{2 p-2}{p \beta}+\frac{2+\kappa}{p}}}{|1-z|^{\left(1+\frac{1}{p}\right)\left(\frac{2 p-2}{\beta}+2\right)}\left|\log \frac{2 e}{1-z}\right|^{\frac{2 p-2}{p \beta}+\frac{2}{p}}} \\
& \times\left|1-\frac{1}{\log \frac{2 e}{1-z}}\right|^{\frac{2 p-2}{\beta}+2} d m(z) \\
& \leq C \int_{\mathbb{B}_{\eta}}\left(1-|z|^{2}\right)^{\frac{2 p-2}{\beta}} \frac{\left|\log \frac{2 e}{1-z}\right|^{\frac{\kappa}{p}}}{|1-z|^{\frac{2 p-2}{\beta}+2-\frac{\kappa}{p}}} d m(z) \\
& \leq C \int_{0}^{1}(1-r)^{-1+\frac{\kappa}{p}}\left(\log \frac{2 e}{1-r}\right)^{\frac{\kappa}{p}} d r<\infty .
\end{aligned}
$$

This finishes the proof.

\section{REFERENCES}

[1] J. M. Anderson, J. Clunie and Ch. Pommerenke, On Bloch functions and normal functions, J. Reine Angew. Math. 270 (1974), 12-37. MR0361090 (50:13536)

[2] A. Baernstein II, D. Girela and J. A. Peláez, Univalent functions, Hardy spaces and spaces of Dirichlet type, Illinois J. Math. 48, n. 3 (2004), 837-859. MR2114254 (2005i:30085)

[3] J. J. Donaire, D. Girela and D. Vukotić, On univalent functions in some Möbius invariant spaces, J. Reine Angew. Math. 553 (2002), 43-72. MR1944807 (2003k:30076)

[4] P. Duren, Theory of $H^{p}$ Spaces, Academic Press, New York-London, 1970. MR 0268655 $(42: 3552)$

[5] P. L. Duren, Univalent Functions, Springer-Verlag, New York, Berlin, Heidelberg, Tokyo, 1983. MR 708494 (85j:30034)

[6] L. Hörmander, Notions of convexity, Progress in Mathematics, vol. 127, Birkhäuser Boston Inc., Boston, MA, 1994. MR.1301332 (95k:00002)

[7] M. Pavlović, Introduction to function spaces on the disk, Posebna Izdanja [Special Editions], vol. 20, Matematički Institut SANU, Beograd, 2004. MR2109650 (2006d:30001)

[8] Ch. Pommerenke, Univalent Functions, Vandenhoeck und Ruprecht, Göttingen, 1975. MR0507768 (58:22526)

[9] Ch. Pommerenke, Boundary Behaviour of Conformal Maps, Springer-Verlag, Berlin, 1992. MR1217706 (95b:30008)

[10] H. Prawitz, Uber Mittelwerte analytischer Funktionen, Ark. Mat. Astr. Fys. 20 A (1927), $1-12$.

Miroslav Pavlović, MatematičKi fakultet, Studentski trg 16, 11001 Belgrade, P.P. 550 , SERBIA

E-mail address: pavlovic@matf.bg.ac.yu

Departamento de Análisis Matemático, Universidad de Málaga, Campus de Teatinos, 29071 Málaga, Spain

E-mail address: japelaez@uma.es 